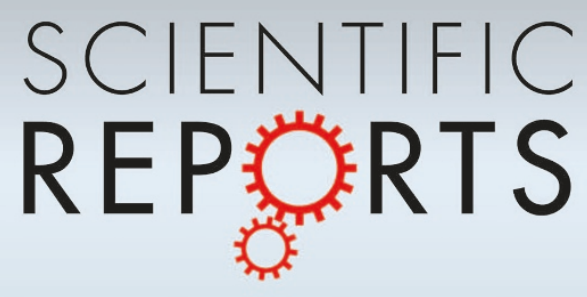

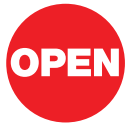

SUBJECT AREAS:

SURFACES, INTERFACES AND THIN FILMS

BIOPHYSICAL METHODS

SUPRAMOLECULAR ASSEMBLY

MEMBRANE STRUCTURE AND

ASSEMBLY

Received

3 September 2012

Accepted

6 March 2013

Published

27 March 2013

Correspondence and requests for materials should be addressed to M.L.G. (mlgee@ unimelb.edu.au) or A.H.A.C. (aclayton@ swin.edu.au)

\section{Imaging the action of antimicrobial peptides on living bacterial cells}

\author{
Michelle L. Gee', Matthew Burton', Alistair Grevis-James', Mohammed Akhter Hossain², Sally McArthur', \\ Enzo A. Palombo ${ }^{4}$, John D. Wade ${ }^{2} \&$ Andrew H. A. Clayton ${ }^{5}$
}

${ }^{1}$ School of Chemistry, University of Melbourne, Parkville, Victoria 3010, Australia, ${ }^{2}$ Howard Florey Institute, University of Melbourne, Parkville, Victoria 3010, Australia, ${ }^{3}$ Industrial Research Institute Swinburne, Faculty of Engineering and Industrial Sciences, Swinburne University of Technology, Hawthorn, Victoria 3122 , Australia, ${ }^{4}$ Faculty of Life and Social Sciences, Swinburne University of Technology, Hawthorn, Victoria 3122, Australia, ${ }^{5}$ Centre for Micro-Photonics, Faculty of Engineering and Industrial Sciences, Swinburne University of Technology, Hawthorn, Victoria 3122 , Australia.

Antimicrobial peptides hold promise as broad-spectrum alternatives to conventional antibiotics. The mechanism of action of this class of peptide is a topical area of research focused predominantly on their interaction with artificial membranes. Here we compare the interaction mechanism of a model antimicrobial peptide with single artificial membranes and live bacterial cells. The interaction kinetics was imaged using time-lapse fluorescence lifetime imaging of a fluorescently-tagged melittin derivative. Interaction with the synthetic membranes resulted in membrane pore formation. In contrast, the interaction with bacteria led to transient membrane disruption and corresponding leakage of the cytoplasm, but surprisingly with a much reduced level of pore formation. The discovery that pore formation is a less significant part of lipid-peptide interaction in live bacteria highlights the mechanistic complexity of these interactions in living cells compared to simple artificial systems.

$\mathrm{T}$ he development of antibiotics is arguably one of humanity's greatest achievements, contributing to significantly increased life expectancy compared to past generations ${ }^{1}$. Our ever-growing dependence on existing conventional antibiotics has resulted in the evolution of antibiotic resistant superbug strains that adapt and become resistant at a rate much faster than our ability to design new $\mathrm{drugs}^{2}$. Antimicrobial peptides are promising therapeutic alternatives ${ }^{3-5}$. They are a natural part of an organism's defense system that can selectively kill invading bacteria without interfering with cells from the host organism ${ }^{6-8}$.

The potential offered by antimicrobial peptides as therapeutic alternatives to current antibiotics has led to a vast literature aimed at understanding how antimicrobial peptides interact with cell membranes. Predominantly, these studies used model membrane systems, such as supported lipid bilayers, vesicles and lamellae and show that many antimicrobial peptides are able lyse biomembranes and form membrane pores via mechanisms such as barrel stave and toroidal pore ${ }^{9-13}$. So it is commonly assumed that antimicrobial peptides kill bacterial cells via similar pore formation mechanisms that lead to cytoplasmic leakage. However there are only a handful of papers that explore lipid-peptide interactions in living cells ${ }^{14-24}$.

To the best of our knowledge, there are no published works specifically aimed at measuring the interaction mechanism of antimicrobial peptides with live bacteria. Here, we address this paucity. We have used time-lapse fluorescence lifetime imaging microscopy (FLIM) to image the interaction kinetics of a model antimicrobial peptide with an artificial membrane system and with live bacteria. Our model antimicrobial peptide is a derivative of melittin (melittin K14) ${ }^{25}$. The polarity-sensitive fluorescent probe AlexaFluor-430 is covalently grafted at the $\mathrm{K} 14$ residue $^{26}$. Melittin is one of the most widely studied antimicrobial peptides ${ }^{27,28}$ and is known to lyse membranes and form pores in artificial membrane systems ${ }^{29}$. Our melittin derivative behaves similarly $y^{25,26}$.

We imaged the interaction kinetics of the fluorescently labeled melittin K14 with giant unilamellar 1,2dipalmitoyl-sn-glycero-3-phosphatidylchloine (DPPC) vesicles and with live E. coli transfected with e-GFP. Our data yield the surprising result that the lytic antimicrobial peptide leads to insignificant pore formation in live bacteria, compared with the artificial membrane system. In spite of this, there is rapid uptake of the peptide by the bacterial cell and concomitant leakage of the cell cytoplasm. This strongly suggests that the mechanism of lipid-peptide interaction in live bacteria is a dynamic process that causes transient disruption of the cell membrane without destroying its structural integrity. 


\section{Results}

To determine the interaction kinetics of our antimicrobial peptide (AlexaFluor 430-labelled melittin K-14) with a model membrane system (DPPC GUVs) and bacteria we employed time-lapse fluorescence lifetime imaging. To interpret our data, we have drawn on our previous work where we have shown that the fluorescence lifetime of the melittin derivative is sensitive to the polarity of its microenvironment and is quenched upon pore formation ${ }^{25}$. A representative fluorescence intensity image of a DPPC giant unilamellar vesicle, with the melittin derivative in solution, is shown in Figure 1. It is clear that the peptide associates with the model membrane, as evidenced by the increased fluorescence intensity distribution near the periphery of the GUV.

To monitor the interaction kinetics of the peptide with the model membrane we recorded fluorescence lifetime images as a function of time after peptide addition. This gives localized net lifetimes at regions of interest that have contributions from different peptide states: peptide free in solution, peptide associated with the membrane, and peptide participating in pore formation ${ }^{26}$. To facilitate the deconvolution of net lifetime into these different peptide states, we transformed the data from our selected region of interest (the GUV) into phasor space ${ }^{30-32}$, where the phase and modulation lifetime values ( $\tau_{\varphi}$ and $\tau_{\mathrm{m}}$, respectively) are represented by a single point on the complex plane. In other words, $\mathrm{x}=\operatorname{m\operatorname {cos}\varphi }$ and $\mathrm{y}=\operatorname{m\operatorname {sin}\varphi }$, where $m$ is the modulation and $\varphi$ is the phase. Figure $2 \mathrm{~A}$ summarizes the temporal progression of the net fluorescence lifetime in phasor space (denoted by crosses). The pure component phasor values for free peptide (black square) and peptide membrane pore (red circle) are also marked. It is clear from the trajectory that as time progresses, the peptide converts from a free-like peptide state and approaches the membrane pore state. The corresponding fractional contribution of these different peptide states as a function of time are represented in Figure 2B. As time progresses there is an increase in peptide membrane pore states at the expense of un-aggregated peptide, plateauing to a high proportion (65\%) of membrane pores. This is consistent with the conventional view that melittin forms pores in synthetic membranes.

Figure 3 shows the fluorescence intensity and fluorescence lifetime images of live E. coli cells transfected with Green Fluorescent Protein (GFP), prior to the addition of peptide. The intensity image confirms the expected intracellular distribution of the GFP. The lifetime of the fluorescence from the cells is consistent with the expected GFP fluorescence lifetime of $2.6 \mathrm{~ns}^{33}$.

Figure 4 presents a series of fluorescence lifetime images of live $E$. coli cells transfected with Green Fluorescent Protein (GFP), at different times after the addition of the melittin derivative. Of particular

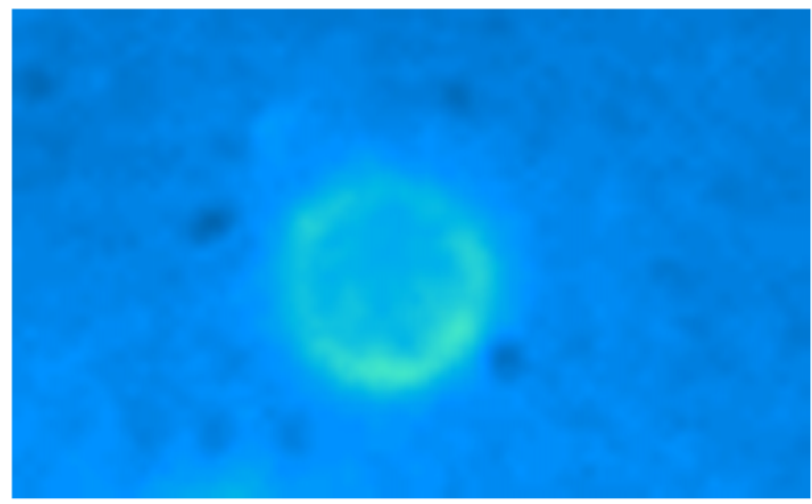

Figure 1 Fluorescence intensity image of a giant-unilamellar vesicle of interest with added melittin derivative. Note the peripheral staining of the vesicle by the fluorescently-labelled melittin derivative showing the association with the membrane. note is the color-change from red to yellow in the background, indicative of a change in the net lifetime. The measured fluorescence lifetime of the background is intermediate between the lifetime of free melittin derivative (ca $3.4 \mathrm{~ns}$ ) and free GFP (ca $2.6 \mathrm{~ns}$ ). This is consistent with rapid leakage of the GFP from cell cytoplasm into the background solution as a result of the melittin derivative interacting with the cells. The corresponding kinetics of the interaction of the melittin derivative with bacteria was investigated by time-lapse lifetime imaging of selected individual bacterial cells, in the same manner as used for the model artificial membranes. The phasor trajectory of the melittin derivative interacting with bacterial cells (Figure 5A) was distinctly different to the trajectory for interaction with model membranes (Figure 2A). Over time, the phasor in the bacterial cells went from a GFP-like state to a free melittin state. This is evident from examining the cosine-component of the phasor as a function of time, Figure $5 \mathrm{~B}$. The direction of this trajectory is opposite to the direction of the model membrane trajectory. The fractional fluorescence contributions from free peptide, membrane pore peptide and GFP are displayed in Figure 5C. As time progresses, the bacteriaassociated GFP contributes less. This is consistent with leakage of the GFP out of the cytoplasm and into the background solution, as also evidenced by the time-lapse lifetime images in Figure 4. The decrease in GFP fractional contribution is concomitant with an increase in free peptide fractional fluorescence, but it should be emphasized that this is free peptide associated with a bacterial cell, not free peptide in bulk solution. This indicates that GFP leakage is a result of peptide translocation from the extracellular solution into the bacterium, most likely the aqueous environment of the cytoplasm. The most surprising result is that the contribution from membrane pore states is ca $5 \%$ at all times (Figure $5 \mathrm{C}$ ), and relative to peptide states no more than $10 \%$ of fractional fluorescence (Eq. 6). This is in stark contrast with the observations on the synthetic membranes in Figure 2B, where the contribution rose from $30 \%$ soon after the addition of peptide, reaching a steady-state of $65 \%$ after approximately 150 minutes.

\section{Discussion}

The striking result that this work uncovers is the massive contrast in the number of pores formed in artificial membranes compared with membrane pores in live bacterial cells, during peptide-membrane interaction. There is a large body of work that shows very clearly that lytic peptides perforate artificial membranes by forming pores. Our expectation was that we would see something similar for the interaction of peptide with living bacterial cells. But surprisingly, what we have measured is very little pore formation at all, in living bacteria. This leaves us with the question of how GFP leaks out from the cell cytoplasm.

From studies on artificial membranes, three mechanisms for membrane disruption or pore formation have been proposed. These are the carpet, barrel stave and toroidal pore models ${ }^{34-37}$. The barrel stave and toroidal pore models are both membrane pore models, but differ in the suggested structure of the pore. In the carpet model, the interaction of peptide with the membrane results in permanent membrane disruption. None of these models can account for our observations on $E$. coli. The fraction of pore states is very low. Complete disruption of the membrane, as per the carpet model, is not observed. The bacterial cells remain remarkably intact.

Our results suggest a new model is needed to explain peptidemembrane interactions in live bacterial cells. E. coli are Gram negative bacteria with a thin peptidoglycan layer/cell wall and two membranes and the leakage of GFP from both inner and outer membrane requires that peptide can access both membranes. The fact that we do see a small fraction of membrane pores shows that pore formation is possible in the membranes of live bacteria. The cooperativity between peptide and lipid needed to form a large number of permanent pores, as seen in artificial membranes ${ }^{38}$, is probably hindered 

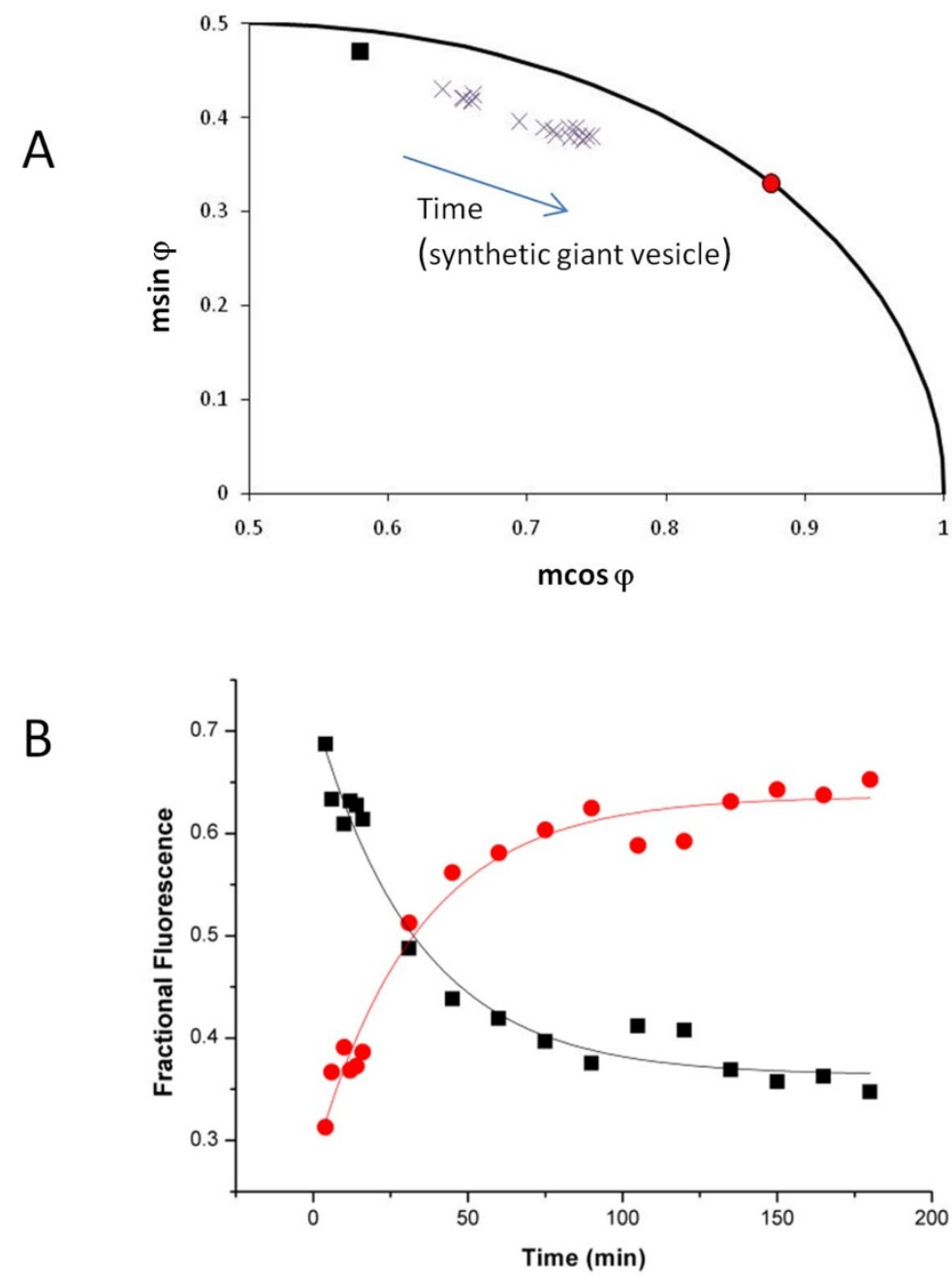

Figure $2 \mid$ Time-lapse FLIM of melittin derivative interacting with a single giant-unilamellar vesicle. (A). Phasor-FLIM representation of the data. $\times$ represents the phasor positions for the time-lapse FLIM data from the melittin derivative averaged over a giant vesicle. The arrow denotes the trajectory of the data as a function of time. $\mathbf{a}$ is the phasor position of the melittin derivative free in buffer solution. represents the phasor position of melittin in a lipidic pore state. (B). Fractional fluorescence from: free melittin, $\mathbf{\square}$; and lipidic pore melittin, $\mathbf{0}$; from a single giant unilamellar vesicle, as a function of time. Fluorescence fractions were determined as described in text. Note the increase in fluorescence from the melittin lipid pore state and the corresponding decrease in the free melittin fluorescence.

by other membrane species (membrane proteins, glycoproteins, lipopolysaccharide etc) on the membranes of bacteria. A logical consequence is that the dynamics of pore formation is altered. It is interesting that the $5 \%-10 \%$ fractional fluorescence of pores observed is seen from almost time zero and remains unchanged. It is tempting to suggest that the pores are kinetic intermediate species
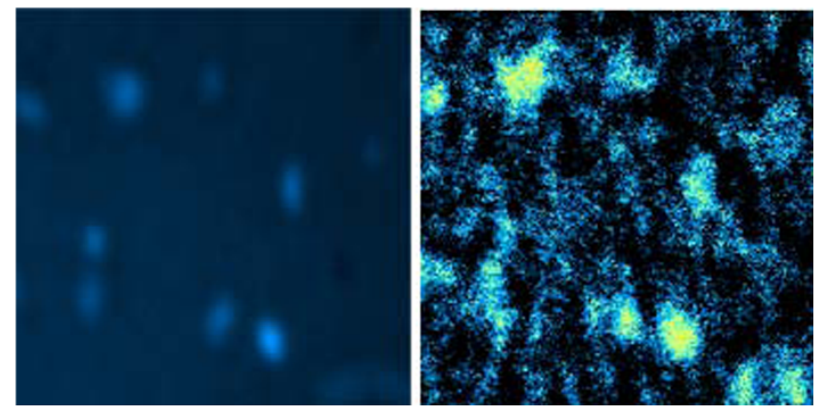

Figure 3 Fluorescence intensity and lifetime images of $E$. coli transfected with GFP prior to addition of peptide. Note the cytoplasmic location of the GFP fluorescence. The lifetime of the GFP in bacteria was $2.6 \mathrm{~ns}$.

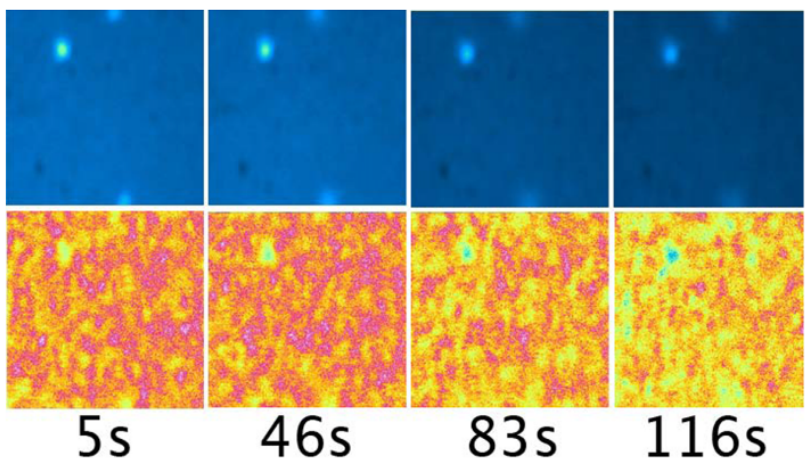

Figure 4 Time-lapse fluorescence intensity and fluorescence lifetime images of $E$. coli transfected with GFP after the addition of peptide at time zero. Note the red to yellow color transition in the background of the lifetime images, which is consistent with an overall decrease in fluorescence lifetime due to leakage of GFP from the cells. 
A

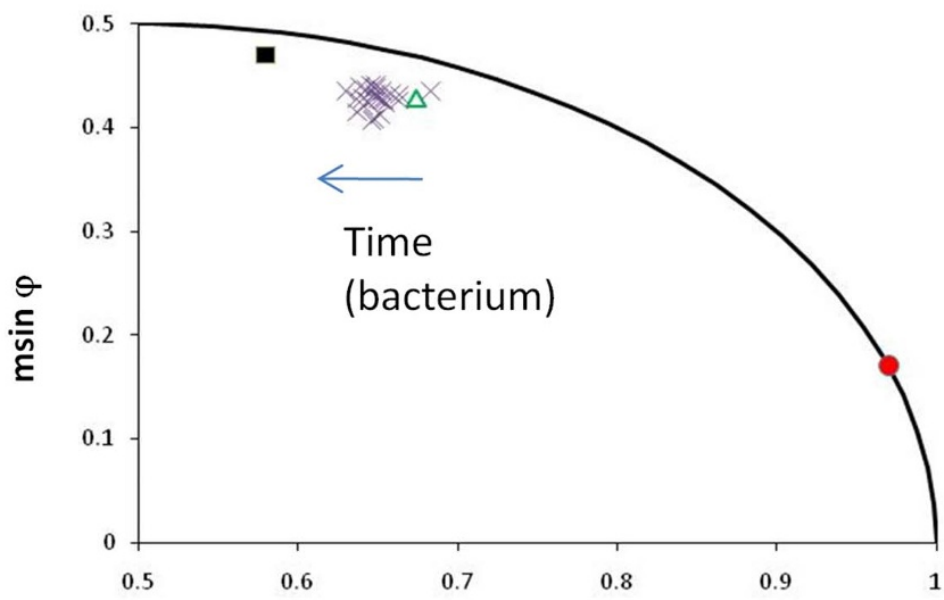

B

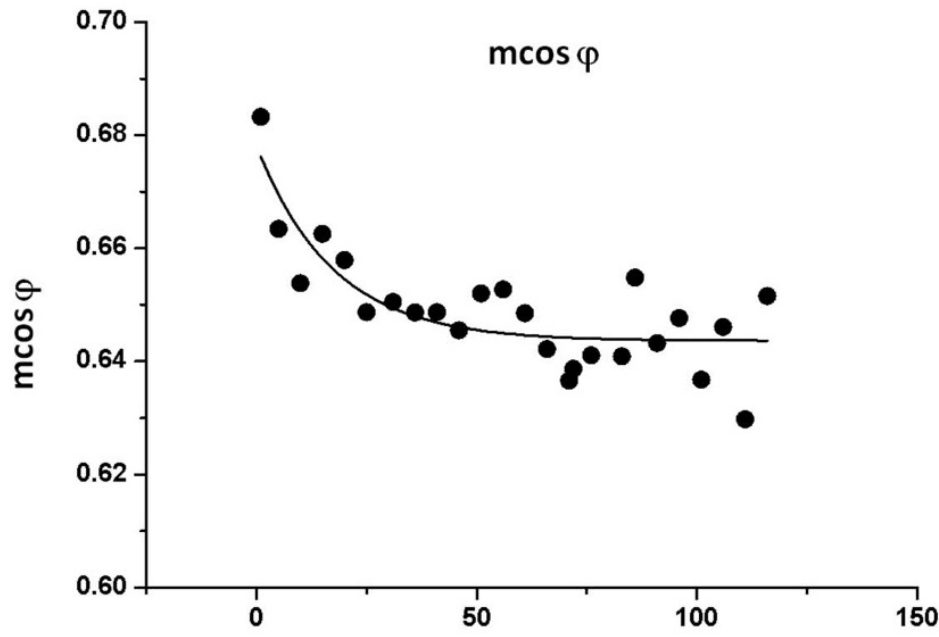

C

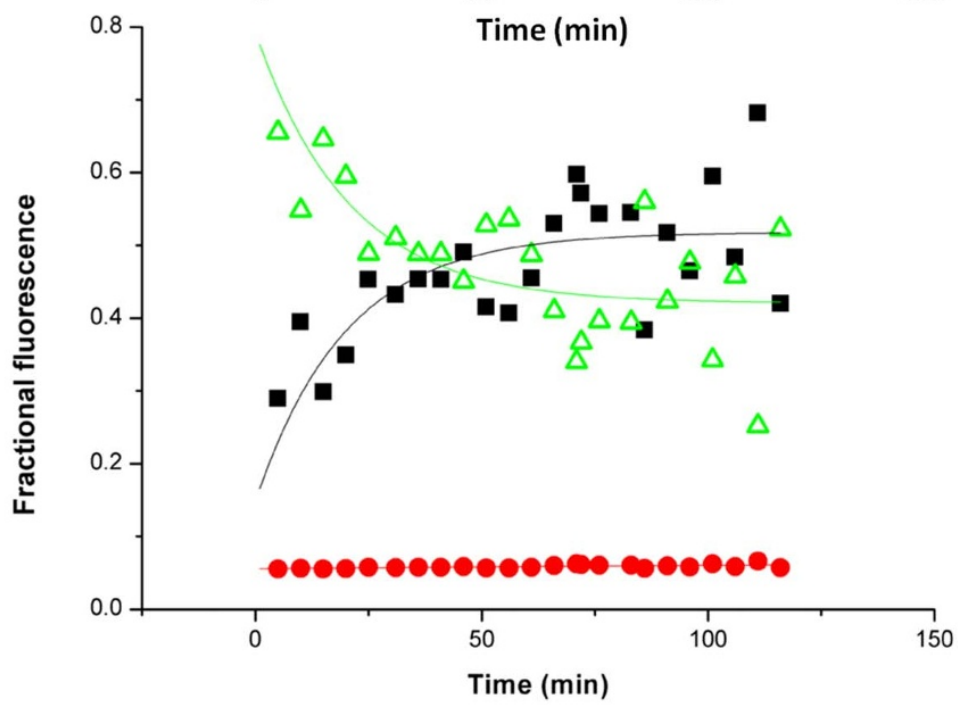

Figure $5 \mid$ Time-lapse FLIM of melittin derivative interacting with a single $\boldsymbol{E}$. coli bacterium containing GFP. (A). Phasor-FLIM representation of the data. $\times$ represents the phasor positions for the time-lapse FLIM data from the bacterial cell. The arrow denotes the trajectory of the data as a function of time. $\mathbf{r e p r e s e n t s ~ t h e ~ p h a s o r ~ p o s i t i o n ~ o f ~ m e l i t t i n ~ d e r i v a t i v e ~ i n ~ b u f f e r . ~ O r e p r e s e n t s ~ t h e ~ p h a s o r ~ p o s i t i o n ~ o f ~ m e l i t t i n ~ i n ~ a ~ l i p i d i c ~ p o r e ~ s t a t e . ~}$ Green triangle represents the phasor position of GFP fluorescence from a bacterial cell. (B). Time trajectory of the cosine-component of the bacterial cell phasor. Solid line is added to guide the eye only. (C). Fractional fluorescence from: free melittin, $\mathbf{\square}$; lipidic pore melittin, $\bigcirc$; and GFP, green triangle; from the bacterial cytoplasm and within the cell wall, as a function of time. Fluorescence fractions were determined using the three-species analysis as described in the text. Note that within the bacterial cell, there is an increase over time in the fractional fluorescence of the melittin derivative and corresponding decrease in the fractional fluorescence of GFP. At all times the lipidic pore state has negligible contribution and remains approximately constant. 


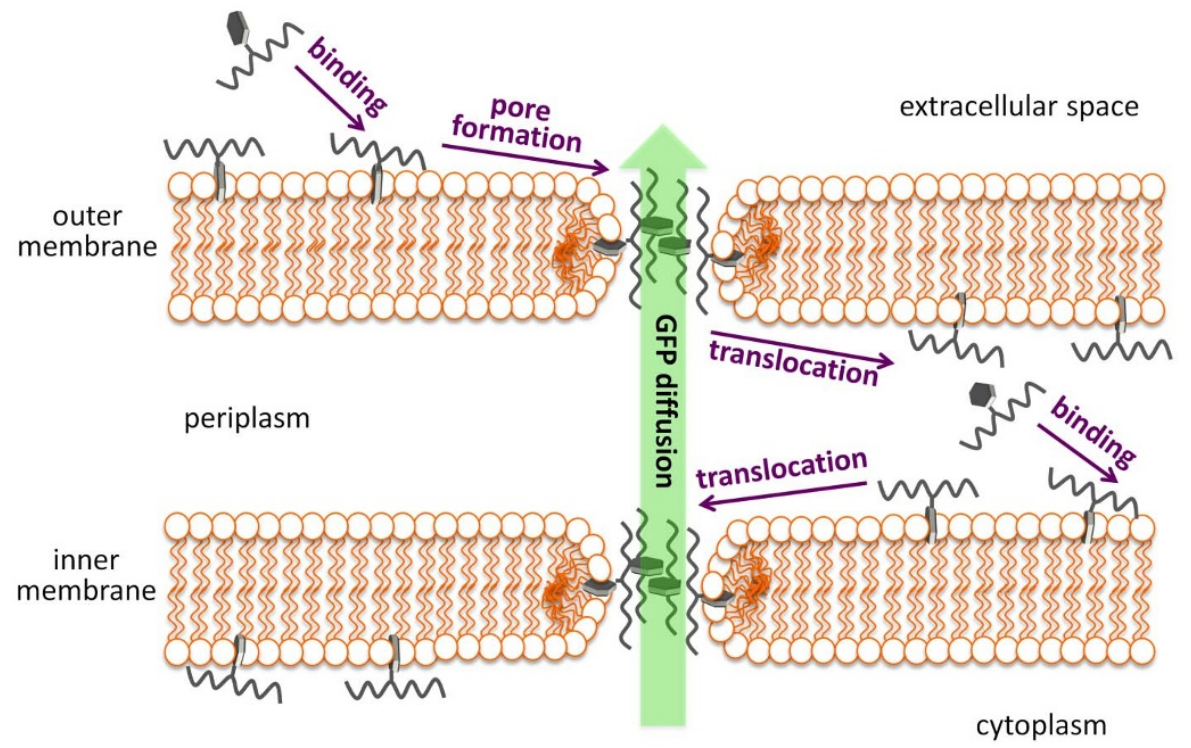

Figure 6 Mechanism for dynamic membrane perturbation by the peptide. In this mechanism the peptide pores are transient species that are in dynamic pseudo-equilibrium with membrane surface states of the peptide. As a result, the peptide molecules can assemble to form transient pores in the outer membrane and then translocate to the inner membrane of gram-negative bacteria. These transient pores are at a low level at steady-state but their dynamic nature allows leakage of cytoplasmic contents from the bacterium.

that, as for chemical intermediates, are transient with a pseudo steady-state concentration, continually forming and breaking up at equal rates (Figure 6). This would constitute significant dynamic perturbation of the bacterial cell membranes. We suggest that it is via these continual local membrane perturbations that GFP is able to rapidly leak out of the cytoplasm.

The results here show that time-lapse lifetime microscopy can provide insight into complex mechanisms that underlie peptidemembrane interactions of cells.

\section{Methods}

Materials. 1,2-dipalmitoyl-sn-glycero-3-phosphatidylcholine (DPPC) was purchased from Avanti Polar Lipids (Alabaster, USA). UV/Vis Spectroscopy grade Chloroform and Methanol were purchased from Merck (Darmstadt, Germany). 4-(2-Hydroxyethyl)-1-piperazineethanesulfonic acid (HEPES, 99\% purity) was purchased from Acros Organics (Geel, Belgium). The fluorescently-labelled melittin derivative, melittin K-14 AlexaFluor-430, was synthesized on a Fmoc-PAL-EG-PS resin, as detailed by Rapson et $\mathrm{al}^{25}$. The AlexaFluor-430 fluorescent label used for the melittin derivative was purchased from Molecular Probes (Carlsbad, USA). All materials were used without further purification. High purity water used in the preparation of all aqueous solutions was generated by a Milli- $\mathrm{Q}^{\mathrm{TM}}$ Ultra Pure Water system or a Milli- $\mathrm{Q}^{\mathrm{TM}}$ Academic system (Millipore Corp., Bedford, USA), and had a resistivity of $18.2 \Omega . \mathrm{m}$.

The GFP expressing plasmid used in this study is a derivative of the vector pGFP (Clontech). It was constructed by taking the NcoI/EcoRI fragment from plasmid pGLO (Bio-Rad) and replacing the equivalent fragment containing the native GFP gene in pGFP. It is believed that the plasmid pGLO is the same as the plasmid pBADGFPuv which has a "fast-folding" mutant form of $\mathrm{GFP}^{39}$. The new plasmid was transformed into competent E. coli JM109. The bacteria were grown overnight at $37^{\circ} \mathrm{C}$ in $\mathrm{LB}$ broth prior to use.

Methods. All glassware was cleaned immediately prior to use by first sonicating for one hour in $10 \%$ Dextran surfactant solution, rinsing with Milli- $\mathrm{Q}^{\mathrm{TM}}$ water followed by soaking for 4 hours in concentrated $(\sim 5 \mathrm{M})$ sodium hydroxide solution then finally rinsing with Milli- $\mathrm{Q}^{\mathrm{TM}}$ water.

All aqueous solutions were $10 \mathrm{mM}$ HEPES that were $\mathrm{pH}$ adjusted to $7.4 \pm 0.05$ and filtered through a $0.22 \mu \mathrm{m}$ Teflon membrane. Melittin derivative solutions were made at a concentration of $9 \mu \mathrm{M}$ in HEPES. All solutions were stored at $4^{\circ} \mathrm{C}$ for no longer than 2 weeks.

Giant Unilamellar Vesicles (GUVs) composed of pure DPPC phospholipid were prepared following the Solvent Evaporation Method as reported by Moscho et $\mathrm{al}^{40}$. Briefly, a $0.1 \mathrm{M}$ solution of DPPC lipid dissolved in chloroform was added to chloroform: methanol mixture (9.8:1 ratio). Filtered HEPES buffer solution was then carefully added to the organic phase. The organic phase removed via vacuum rotary evaporation using a Büchi V-500 Diaphragm Pump operating at $40 \mathrm{rpm}, 40^{\circ} \mathrm{C}$ and $100 \mathrm{mBar}$ minimum pressure. The remaining aqueous GUV suspension was centrifuged at 14,000 rpm (15,300 g) for 10 minutes to remove multilamellar vesicles and lipid debris. The supernatant was collected and stored in glass vials at room temperature $\left(23-26^{\circ} \mathrm{C}\right)$ for no more than two weeks.

A GUV sample was prepared for FLIM by aliquoting $100 \mu \mathrm{L}$ of the GUV supernatant onto a clean glass cover-slip mounted in a Sykes and Moore chamber. The solution was allowed to equilibrate for 45-60 minutes, after which transmitted light microscopy was used to identify a suitable GUV of interest. A sufficient amount of the melittin derivative solution was injected into the sample solution for a global lipid-topeptide (L:P) ratio of $27: 1$. Injections were made as close to the point of interest under the wide field lens as possible to minimize diffusion effects of the fluorescent peptide, since mixing might dislodge the vesicles from their identified locations.

The bacterial samples for FLIM were prepared by depositing $200 \mu \mathrm{L}$ of bacterial suspension onto a clean glass cover-slip mounted in a Sykes and Moore chamber. The bacteria were allowed to adhere for 2 hours. The suspension and any loosely-adhered bacteria were replaced with PBS solution by gentle rinsing. This ensures that the remaining bacteria were surface-immobilized for the duration of the experiment Melittin derivative solution was added to give a final concentration of $9 \mu \mathrm{M}$, which is 2 times the published MIC for melittin/E. coli.

Fluorescence lifetime imaging microscopy (FLIM). All fluorescence lifetime imaging experiments were performed using a LIFA instrument (Lambert Instruments, Leutingwolde, The Netherlands) attached to an inverted microscope (TE2000U, Nikon Inc., Japan). Glass cover slips were used for sample preparation, and observed through a $100 \times$ NA 1.2 oil objective (Nikon Plan-Fluor, Nikon Inc, Japan). The fluorescence excitation source was a $470 \mathrm{~nm}$ LED with a sinusoidal modulation frequency of $40 \mathrm{MHz}$ (Lambert Instruments, Leutingwolde, The Netherlands). Phase and modulation lifetimes were determined by taking a series of 12 phase images of differing phase shift (fluorescence lifetime image stack), utilizing the LI-FLIM software package (Version 1.2.3.11) supporting the LIFA instrument. Photobleaching was corrected through the use of pseudo-random phase ordering in all experiments. The reference used for all lifetime determinations was Rhodamine $6 \mathrm{G}$ (lifetime R6G: $4.1 \mathrm{~ns}$ ). Fluorescence lifetime image stacks were recorded as often as possible after introduction of the peptide for up to 15 minutes (typically 1-4 minute intervals), and then every 15-minute interval beyond that for up to 3 hours. A transmitted light microscopy image was recorded immediately after each fluorescence lifetime image stack acquisition.

The FLIM data were transformed into phasor space, where $\mathrm{x}=\operatorname{mcos} \varphi$ and $\mathrm{y}=\operatorname{msin} \varphi, \mathrm{m}$ is the modulation and $\varphi$ is the phase. To further decompose the fluorescence into fractional states, the fractional fluorescence from free peptide and peptide pore states were computed. For a given phasor, $r(x, y)$, the fractional fluorescence from the peptide pore state, $f_{\text {pore }}$, is given by:

$$
f_{\text {pore }}=\frac{\left|r(x, y)-r(x, y)_{\text {free }}\right|}{\left|r(x, y)_{\text {pore }}-r(x, y)_{\text {free }}\right|}
$$

where $r(x, y)_{\text {free }}$ is the (constant) phasor for free melittin derivative and $r(x, y)_{\text {pore }}$ is the (constant) phasor for peptide pore. These phasors are fixed and used as a point of reference. 
For the bacteria transfected with GFP, there are three states to decompose, GFP fluorescence as well as free peptide and peptide pore. In this case, the observed phasor $(r(x, y)$,$) is the sum of the free peptide, peptide pore and GFP fluorescence phasors,$ weighted by their respective fractional fluorescence contributions $f_{\text {free }}, f_{\text {pore, }}$, and $f_{G F P}$, viz:

$$
r(x, y)=f_{\text {free }} r(x, y)_{\text {free }}+f_{\text {pore }} r(x, y)_{\text {pore }}+f_{G F P} r(x, y)_{G F P}
$$

where

$$
f_{\text {free }}+f_{\text {pore }}+f_{G F P}=1
$$

Substitution of equation (3) into equation (2) and inversion then yields the three desired fractions: $f_{\text {free }}, f_{\text {pore }}$, and $f_{G F P}$.

Because GFP fluorescence can potentially swamp contributions from free and pore-peptide states, it is useful to show that the fraction free and fraction pore states in the absence of GFP fluorescence can be extracted from the data. These values can be determined from the GFP-bacteria using the formulae from equations (4)-(6)

$$
\begin{gathered}
f_{\text {proe }}=\frac{I_{\text {pore }}}{I_{\text {pore }}+I_{G F P}+I_{\text {free }}} \\
f_{\text {free }}=\frac{I_{\text {free }}}{I_{\text {pore }}+I_{\text {GFP }}+I_{\text {free }}}
\end{gathered}
$$

where $I$ is the relative fluorescence intensity for each fluorescent species as specified by the subscript. Combining Equations (4) and (5) to eliminate $I_{G F P}$ gives:

$$
\frac{f_{\text {pore }}}{f_{\text {free }}}=\frac{I_{\text {pore }}}{I_{\text {free }}}=\frac{f_{\text {pore }, \text { no GFP }}}{f_{\text {free, } n \text { G GFP }}}
$$

Equation (6) shows that the ratio of the fractional fluorescence for pore and free states is the same in the absence and presence of GFP.

1. Clardy, J., Fischbach, M. A. \& Currie, C. R. The natural history of antibiotics. Curr Biol 19, R437-441 (2009).

2. Fish, D. N., Piscitelli, S. C. \& Danziger, L. H. Development of resistance during antimicrobial therapy: a review of antibiotic classes and patient characteristics in 173 studies. Pharmacotherapy 15, 279-291 (1995).

3. Giuliani, A. \& Rinaldi, A. C. Beyond natural antimicrobial peptides: multimeric peptides and other peptidomimetic approaches. Cell Mol Life Sci 68, 2255-2266 (2011).

4. Giuliani, A. et al. Antimicrobial peptides: natural templates for synthetic membrane-active compounds. Cell Mol Life Sci 65, 2450-2460 (2008)

5. Hancock, R. E. \& Lehrer, R. Cationic peptides: a new source of antibiotics. Trends Biotechnol 16, 82-88 (1998).

6. Andreu, D. \& Rivas, L. Animal antimicrobial peptides: an overview. Biopolymers 47, 415-433 (1998).

7. Duclohier, H., Molle, G. \& Spach, G. Antimicrobial peptide magainin I from Xenopus skin forms anion-permeable channels in planar lipid bilayers. Biophys 56, 1017-1021 (1989).

8. Zasloff, M. Magainins, a class of antimicrobial peptides from Xenopus skin: isolation, characterization of two active forms, and partial cDNA sequence of a precursor. Proc Natl Acad Sci U S A 84, 5449-5453 (1987).

9. He, K., Ludtke, S. J., Huang, H. W. \& Worcester, D. L. Antimicrobial peptide pores in membranes detected by neutron in-plane scattering. Biochemistry 34, 15614-15618 (1995).

10. Huang, H. W. Peptide-lipid interactions and mechanisms of antimicrobial peptides. Novartis Found Symp 225, 188-200; discussion 200-186 (1999).

11. Wu, Y., He, K., Ludtke, S. J. \& Huang, H. W. X-ray diffraction study of lipid bilayer membranes interacting with amphiphilic helical peptides: diphytanoyl phosphatidylcholine with alamethicin at low concentrations. Biophys J 68, 2361-2369 (1995).

12. Ludtke, S. J., He, K., Wu, Y. \& Huang, H. W. Cooperative membrane insertion of magainin correlated with its cytolytic activity. Biochim Biophys Acta 1190, 181-184 (1994).

13. Huang, H. W. \& Wu, Y. Lipid-alamethicin interactions influence alamethicin orientation. Biophys J 60, 1079-1087 (1991).

14. Leptihn, S. et al. Single molecule resolution of the antimicrobial action of quantum dot-labeled sushi peptide on live bacteria. BMC Biol 7, 22 (2009).

15. Duchardt, F., Fotin-Mleczek, M., Schwarz, H., Fischer, R. \& Brock, R. A comprehensive model for the cellular uptake of cationic cell-penetrating peptides. Traffic 8, 848-866 (2007).

16. Park, C. B., Kim, H. S. \& Kim, S. C. Mechanism of action of the antimicrobial peptide buforin II: buforin II kills microorganisms by penetrating the cell membrane and inhibiting cellular functions. Biochem Biophys Res Commun 244 253-257 (1998).

17. Liang, J. F. \& Kim, S. C. Not only the nature of peptide but also the characteristics of cell membrane determine the antimicrobial mechanism of a peptide. J Pept Res 53, 518-522 (1999).

18. Lazarev, V. N. et al. Induced expression of melittin, an antimicrobial peptide, inhibits infection by Chlamydia trachomatis and Mycoplasma hominis in a HeLa cell line. Int J Antimicrob Agents 19, 133-137 (2002).
19. Chen, Y. L. et al. Novel cationic antimicrobial peptide GW-H1 induced caspasedependent apoptosis of hepatocellular carcinoma cell lines. Peptides 36, 257-265 (2012).

20. Mangoni, M. L. et al. Effects of the antimicrobial peptide temporin L on cell morphology, membrane permeability and viability of Escherichia coli. Biochem $J$ 380, 859-865 (2004).

21. Fantner, G. E., Barbero, R. J., Gray, D. S. \& Belcher, A. M. Kinetics of antimicrobial peptide activity measured on individual bacterial cells using high-speed atomic force microscopy. Nat Nanotechnol 5, 280-285 (2010).

22. Pandey, B. K. et al. Cell-selective lysis by novel analogues of melittin against human red blood cells and Escherichia coli. Biochemistry 49, 7920-7929 (2010).

23. Xing, B. et al. Multifunctional divalent vancomycin: the fluorescent imaging and photodynamic antimicrobial properties for drug resistant bacteria. Chem Commun (Camb) 47, 1601-1603 (2011).

24. Liu, F. et al. Lipopolysaccharide neutralizing peptide-porphyrin conjugates for effective photoinactivation and intracellular imaging of gram-negative bacteria strains. Bioconjug Chem 23, 1639-1647 (2012).

25. Rapson, A. C. et al. Structural dynamics of a lytic peptide interacting with a supported lipid bilayer. Biophys J 100, 1353-1361 (2011)

26. Ningsih, Z., Hossain, M. A., Wade, J. D., Clayton, A. H. \& Gee, M. L. Slow insertion kinetics during interaction of a model antimicrobial peptide with unilamellar phospholipid vesicles. Langmuir 28, 2217-2224 (2012).

27. Raghuraman, H. \& Chattopadhyay, A. Melittin: a membrane-active peptide with diverse functions. Biosci Rep 27, 189-223 (2007).

28. Dempsey, C. E. The actions of melittin on membranes. Biochim Biophys Acto 1031, 143-161 (1990).

29. Smith, R. et al. Structure and orientation of the pore-forming peptide, melittin, in lipid bilayers. J Mol Biol 241, 456-466 (1994).

30. Clayton, A. H., Hanley, Q. S. \& Verveer, P. J. Graphical representation and multicomponent analysis of single-frequency fluorescence lifetime imaging microscopy data. J Microsc 213, 1-5 (2004).

31. Digman, M. A., Caiolfa, V. R., Zamai, M. \& Gratton, E. The phasor approach to fluorescence lifetime imaging analysis. Biophys J 94, L14-16 (2008).

32. Redford, G. I. \& Clegg, R. M. Polar plot representation for frequency-domain analysis of fluorescence lifetimes. J Fluoresc 15, 805-815 (2005).

33. Subramaniam, V., Hanley, Q. S., Clayton, A. H. \& Jovin, T. M. Photophysics of green and red fluorescent proteins: implications for quantitative microscopy. Methods Enzymol 360, 178-201 (2003).

34. Shai, Y. \& Oren, Z. From "carpet" mechanism to de-novo designed diastereomeric cell-selective antimicrobial peptides. Peptides 22, 1629-1641 (2001).

35. Yang, L., Harroun, T. A., Weiss, T. M., Ding, L. \& Huang, H. W. Barrel-stave model or toroidal model? A case study on melittin pores. Biophys J 81, 1475-1485 (2001).

36. Laver, D. R. The barrel-stave model as applied to alamethicin and its analogs reevaluated. Biophys J 66, 355-359 (1994)

37. Park, S. C. et al. Investigation of toroidal pore and oligomerization by melittin using transmission electron microscopy. Biochem Biophys Res Commun 343, 222-228 (2006).

38. Huang, H. W. Action of antimicrobial peptides: two-state model. Biochemistry 39, 8347-8352 (2000).

39. Crameri, A., Whitehorn, E. A., Tate, E. \& Stemmer, W. P. Improved green fluorescent protein by molecular evolution using DNA shuffling. Nat Biotechnol 14, 315-319 (1996)

40. Moscho, A., Orwar, O., Chiu, D. T., Modi, B. P. \& Zare, R. N. Rapid preparation of giant unilamellar vesicles. Proc Natl Acad Sci U S A 93, 11443-11447 (1996).

\section{Acknowledgements}

MLG and AHAC acknowledge the Australian Research Council for funding support (grant no. DP110100164). Matthew Burton is a recipient of an Australian Postgraduate Research Award.

\section{Author contributions}

M.L.G., M.B., A.G.J., M.A.H. and A.H.A.C. performed experiments and/or analysed data; S.M., E.A.P., J.D.W., M.L.G. and A.H.A.C. designed the research; M.L.G. and A.H.A.C wrote the paper.

\section{Additional information}

Competing financial interests: The authors declare no competing financial interests.

License: This work is licensed under a Creative Commons

Attribution-NonCommercial-NoDerivs 3.0 Unported License. To view a copy of this license, visit http://creativecommons.org/licenses/by-nc-nd/3.0/

How to cite this article: Gee, M.L. et al. Imaging the action of antimicrobial peptides on living bacterial cells. Sci. Rep. 3, 1557; DOI:10.1038/srep01557 (2013). 